2. W. Hahn, Theorie und Anwendung der direkten Methode von Ljapunov, Ergebnisse der Mathematik und ihrer Grenzgebiete, Neue Folge, Heft 22, Springer-Verlag, 1959

3. R. E. Kalman, Y. C. Ho and K. S. Narendra, Controllability of linear dynamical systems, Contributions to Differential Equations, Vol. 1, Interscience, 1963, pp. 189-213

4. J. LaSalle and S. Lefschetz, Stability by Liapunov's direct method with applications, Academic Press, 1961

5. J. P. LaSalle, The time optimal control problem, Contributions to the Theory of non-linear Oscillations, vol. 5, Princeton University Press, 1960, pp. 1-24

6. W. T. Reid, Riccati matrix differential equations and non-oscillation criteria for associated linear differential systems, Pacific J. Math. 13 (1963) 665-685

7. W. T. Reid, Principal solutions of non-oscillatory linear differential systems, to appear in J. Math. Anal. and Appl.

\title{
CONDITIONS FOR THE CAUSALITY OF NONLINEAR OPERATORS DEFINED ON A FUNCTION SPACE*
}

By I. W. SANDBERG (Bell Telephone Laboratories, Inc., Murray Hill, New Jersey)

Abstract. This note considers nonlinear operators $T$ with both range and domain subsets of the space of complex $n$-vector-valued functions of the real variable $t$ for $-\infty<t<\infty$. Conditions, in which energy-type quantities play a key role, are presented under which $T$ is causal in the following sense:

Let $D(T)$ denote the domain of $T$ and let $t_{0}=\sup \left\{t^{\prime} \mid\right.$ for all $f \varepsilon D(T), f(t)=0$ for almost all $\left.t<t^{\prime}\right\}$. Then $T$ is causal if for an arbitrary $\delta>t_{0}, T f=T g$ a.e. on $\left(t_{0}, \delta\right)$ whenever $f$ and $g$ belong to $D(T)$ and $f=g$ a.e. on $\left(t_{0}, \delta\right)$.

1. Notation. Let $\mathfrak{H C}_{n}$ denote the space of complex measurable $n$-vector-valued functions of the real variable $t$ for $-\infty<t<\infty$. The complex-conjugate transpose of an arbitrary $f \varepsilon \mathcal{F}_{n}$ is written as $f^{*}$. With $g$ and $h$ arbitrary elements of $\mathfrak{H}_{n}$, and $x$ either an arbitrary real number or $\infty$, let $\langle g, h ; x\rangle$ denote

$$
\int_{-\infty}^{x} g^{*} h d t
$$

and let

$$
\|g ; x\|=(\langle g, g ; x\rangle)^{1 / 2} .
$$

The symbol $R$ denotes the set of real-valued functions and

$$
\mathfrak{L}_{2 n}=\left\{f \mid f \varepsilon \mathcal{K}_{n},\langle f, f ; \infty\rangle<\infty\right\} .
$$

If $f \varepsilon \mathfrak{F C}_{n}$, and $x<\infty$, then $f_{x}$ is defined by

$$
\begin{aligned}
f_{x}=f, & t \leq x \\
f_{x}=0, & t>x .
\end{aligned}
$$

2. Introduction. The external properties of a physical system can frequently be characterized by an operator relation of the form

$$
g=T f,
$$

*Received February 26, 1964; revised manuscript received July 2, 1964. 
in which $g$ and $f$ are $n$-vector-valued functions of $t$ such that $\operatorname{Re}\left(g^{*} f\right)$ represents the total instantaneous power entering the system. For example, for an electro-mechanical system, the $j$ th component of $f$ might be the voltage or force at the $j$ th accessible point of the system, in which case $g$ would be the corresponding $n$-vector of currents and velocities.

The notions of energy and causality play a central role in the study of the properties of physical systems. It is of interest therefore to consider the relation between energy type properties of a general operator $T$ and the causality or lack of causality of $T$.

In order to be more specific, it is convenient to introduce the following definitions in which $T$ denotes an arbitrary single-valued (not necessarily linear) operator with range and domain contained in $\mathfrak{H C}_{n}$. The domain of $T$ is denoted by $D(T)$.

Definition 1: We shall say that $T$ is passive if for all real $x$ and all $f \varepsilon D(T),\langle T f, f ; x\rangle$ is well-defined and finite, and $\operatorname{Re}\langle T f, f ; x\rangle \geq 0$.

Definition 2. Let $t_{0}=\sup \left\{t^{\prime} \mid\right.$ for all $f \varepsilon D(T), f(t)=0$ for almost all $\left.t<t^{\prime}\right\}$, with the understanding that $t_{0}=-\infty$ if for every $t^{\prime}$ there exist a positive-measure subset $\delta$ of $\left(-\infty, t^{\prime}\right)$, and an $f \varepsilon \mathbb{D}(T)$, such that $f(t) \neq 0$ for $t \varepsilon \mathcal{S}$.

It is assumed throughout that $t_{0}<\infty$.

Definition 3. We shall say that $T$ is causal if for an arbitrary $\delta>t_{0}, T f=T g$ a.e. on $\left(t_{0}, \delta\right)$ whenever $f, g \varepsilon D(T)$ and $f=g$ a.e. on $\left(t_{0}, \delta\right)$.

In their interesting paper, ${ }^{1}$ Youla, Castriota, and Carlin [1] take $t_{0}=-\infty$ in the definition above and prove that a sufficient condition for a linear passive $T$ to be causal is that there exist in $\mathscr{D}(T) n$ elements $v_{i}(j=1,2, \cdots, n)$ such that the $v_{j}(t)$ are linearly independent for almost every $t$ in $(-\infty, \infty)$.

In this note we present conditions under which nonlinear operators are causal. For example, a direct corollary of our main result asserts that if $D(T)=\mathfrak{L}_{2 n}$ and $T$ maps $\mathfrak{L}_{2 n}$ into itself such that there exists a functional $M(f)$ defined on $\mathfrak{L}_{2 n}$ with the properties that $M(f)=M(g)$ if $f=g$ a.e., $M(\beta f) \rightarrow 0$ as $\beta \rightarrow 0$, and $\|T h-T k ; \infty\| \leq M(h-k)$ for all $h, k \varepsilon \mathscr{L}_{2 n}$, then $T$ is causal if and only if

$$
\operatorname{Re}\langle T h-T k, h-k ; x\rangle \geq-M\left(h_{x}-k_{x}\right)\|h-k ; x\|
$$

for all real $x$ and all $h, k \varepsilon \mathfrak{L}_{2 n}$.

A physical interpretation of some of the results is implied by Theorem 3, in which attention is focused on the "weak linearization" of $T$ about an arbitrary element of its domain. For example, with $T$ a mapping of $\aleph_{2 n}$ into itself, if every weak linearization of $T$ yields a passive operator, then Theorem 3 asserts that $\operatorname{Re}\langle T h-T k, h-k ; x\rangle \geq 0$ for all $h, k \varepsilon \mathfrak{L}_{2 n}$. Thus, it follows from our results that if $D(T)=\mathfrak{L}_{2 n}$ and $T$ maps $\mathfrak{L}_{2 n}$ into itself such that there exists a functional $M(f)$ with the properties stated above, $T$ is causal if the weak linearization of $T$ about an arbitrary element of $\mathcal{L}_{2 n}$ yields a passive operator.

3. Results. Our principal result is the following theorem.

Theorem 1. Let $T$ be a (not necessarily linear) mapping into $\mathfrak{H}_{n}$, with domain any subset $\mathscr{D}(T)$ of $\mathcal{H C}_{n}$ such that:

(1) $\langle T h-T k, h-k ; x\rangle$ is well-defined and finite for all real $x$ and all $h, k \varepsilon \mathfrak{D}(T)$.

(2) $\operatorname{Re}\langle T h-T k, h-k ; x\rangle \geq U(h-k ; x)$ for all real $x$ and all $h, k \varepsilon \mathfrak{D}(T)$, where $U(f ; x)$ is a functional defined on $\mathcal{E}(T)=\{f \mid f=h-k$ with $h, k \varepsilon D(T)\}$ such that

(a) $U(f ; x)=U(g ; x)$ if $f, g \varepsilon \varepsilon(T)$ and $f=g$ for almost all $t<x$.

(b) $\beta^{-1} U(\beta f ; x) \rightarrow 0$ as $\beta \rightarrow 0$, with $\beta$ real or pure imaginary. 
(3) for any $v \varepsilon \mathbb{D}(T)$, there exists a positive number $\sigma_{0}$ and a subset $\left\{v_{i}, 1 \leq j \leq n\right\}$ of $\mathfrak{H C}_{n}$ such that

(a) the $v_{i}(t)$ are linearly independent for almost all $t \varepsilon\left(t_{0}, \infty\right)$

(b) $\left\{v+\sigma v_{i}, 1 \leq j \leq n\right\} \subset D(T)$ for every real or pure imaginary number $\sigma$ such that $|\sigma| \leq \sigma_{0}$

(c) for all real $x$, and $\sigma$ real or pure imaginary, $\left\langle T\left(v+\sigma v_{i}\right)-T(v), v_{i} ; x\right\rangle \rightarrow 0$ as $\sigma \rightarrow 0$.

Then $T$ is causal.

Proof. Assume that $f, g \varepsilon \mathbb{D}(T)$ and that $f=g$ a.e. on $\left(t_{0}, \delta\right)$, where $\delta$ is an arbitrary real number such that $\delta>t_{0}$. Let $\left\{v_{i}, 1 \leq j \leq n\right\}$ have the properties stated in the theorem, with $v=g$.

Consider the following inequality which, according to the second hypothesis, is valid for all real $x$

$$
\operatorname{Re}\left\langle T\left(g+\sigma v_{i}\right)-T g-[T f-T g], g-f+\sigma v_{j} ; x\right\rangle \geq \mathcal{L}^{\prime}\left(g-f+\sigma v_{i} ; x\right)
$$

Because $f=g$ a.e. on $(-\infty, \delta)$, it follows that for $x<\delta$ and every real or pure imaginary $\sigma$ such that $|\sigma| \leq \sigma_{0}$

$$
\operatorname{Re}\left\{\sigma\left\langle T\left(g+\sigma v_{i}\right)-T(g), v_{i} ; x\right\rangle\right\}-\operatorname{Re}\left\{\sigma\left\langle T f-T g, v_{i} ; x\right\rangle\right\} \geq U\left(\sigma v_{i} ; x\right) .
$$

This inequality implies that

$$
\left\langle T f-T g, v_{i} ; x\right\rangle=0 \text { for } x \varepsilon(-\infty, \delta),
$$

since otherwise, in view of the assumptions that $\left\langle T\left(g+\sigma v_{j}\right)-T g, v_{i} ; x\right\rangle$ and $\sigma^{-1} U\left(\sigma v_{j} ; x\right)$ approach zero as $\sigma \rightarrow 0$, there would exist an $x \varepsilon(-\infty, \delta)$ and a real or pure imaginary $\sigma$ with $|\sigma| \leq \sigma_{0}$ for which (2) was not satisfied.

It is obvious that the derivative of the left-hand side of (3), with respect to $x$, vanishes for $x \varepsilon(-\infty, \delta)$. Thus

$$
[T f-T g]^{*} v_{i}=0 \quad \text { a.e. on }(-\infty, \delta)
$$

for $j=1,2, \cdots, n$, which, since the $v_{j}(t)$ are linearly independent for almost all $t \varepsilon\left(t_{0}, \infty\right)$, implies that

$$
T f=T g \quad \text { a.e. } \quad \text { on }\left(t_{0}, \delta\right) .
$$

This proves the theorem. ${ }^{\dagger}$

Remarks. It is evident from the proof of Theorem 1 that the conclusion remains valid if $D(T)=\mathscr{R} \cap D(T)$ and hypotheses 2(b), 3(b), and 3(c) are satisfied only for real $\beta$ and real $\sigma$.

Observe that condition (3) of the theorem is satisfied except in rather pathological cases. A very simple example showing that a condition of the type 3(a) is actually needed is given in Ref. 1 .

Theorem 2. Let $T$ be a mapping of $D(T)$, a subset of $\mathfrak{F C}_{n}$, into $\mathfrak{F C}_{n}$ such that the first and third hypotheses of Theorem 1 are satisfied. For arbitrary real $x$, let $f_{x} \varepsilon \mathcal{D}(T)$ whenever $f \varepsilon D(T)$. Suppose that there exists a functional $M(f ; x)$ defined on $\varepsilon(T)=$ $\{f \mid f=h-k$ with $h, k \varepsilon D(T)\}$ such that $M(f ; x)=M(g ; x)$ if $f=g$ a.e., $M(\beta f ; x) \rightarrow 0$

†After Theorem 1 had been proved, the author was informed by F. T. Boesch and D. C. Youla that they had independently obtained, in an unpublished study, a result similar to that to which Theorem 1 reduces for $T$ linear. 
as $\beta \rightarrow 0$, and $\|T h-T k ; x\| \leq M(h-k ; x)$ for all $x$ and all $h, k \varepsilon D(T)$. Suppose also that for all $f \varepsilon \varepsilon(T),\|f ; x\|<\infty$ for $t_{0}<x<\infty$. Then $T$ is causal if and only if

$$
\operatorname{Re}\langle T h-T k, h-k ; x\rangle \geq-M\left(h_{x}-k_{x} ; x\right)\|h-k ; x\|
$$

for all real $x$ and all $h, k \varepsilon \mathscr{D}(T)$.

Proof. Suppose that $T$ is causal. Then

$$
\begin{aligned}
|\operatorname{Re}\langle T h-T k, h-k ; x\rangle| & \leq\|T h-T k ; x\| \cdot\|h-k ; x\| \\
& \leq\left\|T h_{x}-T k_{x} ; x\right\| \cdot\|h-k ; x\| \\
& \leq M\left(h_{x}-k_{x} ; x\right)\|h-k ; x\| .
\end{aligned}
$$

Hence $T$ causal implies that

$$
\operatorname{Re}\langle T h-T k, h-k ; x\rangle \geq-M\left(h_{x}-k_{x} ; x\right)\|h-k ; x\|
$$

for all real $x$ and all $h, k \varepsilon D(T)$. As far as the sufficiency is concerned, observe that the functional $-M\left(h_{x}-k_{x} ; x\right)\|h-k ; x\|$ meets the conditions of the second hypothesis of Theorem 1.

Corollary $2(a)$. Let $\mathcal{K}$ denote either $\mathfrak{L}_{2 n}$ or $R \cap \mathfrak{L}_{2 n}$. Suppose that $T$ is a mapping of $\mathcal{K}$ into itself with the property that there exists a functional $M(f)$ defined on $\mathcal{K}$ such that $M(f)=M(g)$ if $f=g$ a.e., $M(\beta f) \rightarrow 0$ as $\beta \rightarrow 0$, and $\|T h-T k ; \infty\| \leq M(h-k)$ for all $h, k \varepsilon \mathcal{K}$. Then $T$ is causal if and only if

$$
\operatorname{Re}\langle T h-T k, h-k ; x\rangle \geq-M\left(h_{x}-k_{x}\right)\|h-k ; x\|
$$

for all real $x$ and all $h, k \varepsilon \Re$.

Proof: The first and third hypotheses of Theorem 1 are satisfied with $\mathscr{D}(T)=\mathscr{K}$ (for which $t_{0}=-\infty$ ). In particular, for every $v \varepsilon \Re$ we may take $v_{i}$ to be the $n$-vector with the $j$ th component equal to $e^{-|t|}$ and the remaining components equal to zero for all $t$.

With regard to part (c) of the third hypothesis,

$$
\begin{aligned}
\left|\left\langle T\left(v+\sigma v_{j}\right)-T(v), v_{i} ; x\right\rangle\right| & \leq\left\|T\left(v+\sigma v_{i}\right)-T(v) ; x\right\| \cdot\left\|v_{j} ; x\right\| \\
& \leq\left\|T\left(v+\sigma v_{i}\right)-T(v) ; \infty\right\| \cdot\left\|v_{i} ; \infty\right\| \\
& \leq M\left(\sigma v_{i}\right)\left\|v_{i} ; \infty\right\| .
\end{aligned}
$$

Thus for all real $x,\left\langle T\left(v+\sigma v_{i}\right)-T(v), v_{i} ; x\right\rangle \rightarrow 0$ as $\sigma \rightarrow 0$.

Remark. For operators that represent physical systems, it is often the case that there exists a constant $c$ such that $M(f)$ can be taken to be simply $c\|f ; \infty\|$.

In the following theorem it is assumed that $T$ is a mapping of $\mathfrak{L}_{2 n}$ or $R \cap \mathfrak{L}_{2 n}$ into itself and that $T$ possesses a "weak linearization" (as opposed to the "strong linearization" involved, for example, in the definition of the Fréchet differential) about each element of its domain. A special case of the theorem asserts that the condition: $\langle T h-T k, h-k ; x\rangle \geq 0$ for all real $x$, is satisfied if and only if the linearization of $T$ about an arbitrary element of its domain yields a passive operator.

Theorem 3. Let $\mathcal{K}$ denote either $\mathfrak{L}_{2 n}$ or $R \cap \mathfrak{L}_{2 n}$. Suppose that $T$ is a mapping of $\mathcal{K}$ into itself such that for any $f, g \varepsilon \Re$ there exists a linear operator $L_{f}$, which maps $\mathfrak{K}$ into itself and depends continuously on $f$, with the property that

$$
T(f+\lambda g)-T(f)=L_{f}(\lambda g)+r(f, \lambda g),
$$


in which $\lambda$ is a scalar and $\lambda^{-1}\|r(f, \lambda g) ; \infty\| \rightarrow 0$ as $\lambda \rightarrow 0$. Let $U(f ; x)$ be a functional defined on $\Re$ such that for every $f \varepsilon \mathcal{K}$ and every constant $\alpha, U(\alpha f ; x)=|\alpha|^{2} U(f ; x)+$ $V(\alpha, f, x)$, in which $\alpha^{-2} V(\alpha, f, x) \rightarrow 0$ as $\alpha \rightarrow 0$. Then the following two statements are equivalent.

(i) $\operatorname{Re}\left\langle L_{h}(k), k ; x\right\rangle \geq U(k ; x)$ for all real $x$ and all $h, k \varepsilon \mathfrak{K}$

(ii) $\operatorname{Re}\langle T h-T k, h-k ; x\rangle \geq U(h-k ; x)$ for all real $x$ and all $h, k \varepsilon \nVdash$.

Proof. The fact that (ii) implies (i) is an obvious consequence of the fact that (ii) implies that

$$
\operatorname{Re}\left\langle L_{f}(\lambda g), \lambda g ; x\right\rangle+\operatorname{Re}\langle r(f, \lambda g), \lambda g ; x\rangle \geq L^{\prime}(\lambda g ; x)
$$

for all $\lambda$, all real $x$, and all $f, g \varepsilon \mathfrak{K}$. Then

To show that (i) implies (ii), let $y(\alpha)=T[\alpha(h-k)+k]$ with $0 \leq \alpha \leq 1$ and $h, k \varepsilon \mathfrak{K}$

$$
T h-T k=y(1)-y(0)=\int_{0}^{1} y^{\prime}(\alpha) d \alpha,
$$

in which, with $q=\alpha(h-k)+k$,

$$
y^{\prime}(\alpha)=\lim _{\Delta \alpha \rightarrow 0}[y(\alpha+\Delta \alpha)-y(\alpha)] / \Delta \alpha=L_{a}(h-k),
$$

and hence

$$
\begin{aligned}
\operatorname{Re}\langle T h-T k, h-k ; x\rangle & =\operatorname{Re}\left\langle\int_{0}^{1} y^{\prime}(\alpha) d \alpha, h-k ; x\right\rangle \\
& =\int_{0}^{1} \operatorname{Re}\left\langle y^{\prime}(\alpha), h-k ; x\right\rangle d \alpha \\
& =\int_{0}^{1} \operatorname{Re}\left\langle L_{a}(h-k) ; h-k, x\right\rangle d \alpha .
\end{aligned}
$$

If (i) holds, the last integral is at least equal to

$$
\int_{0}^{1} U(h-k ; x) d \alpha=U(h-k ; x) .
$$

This proves the theorem.

Remark: Observe that (i) implies (ii) even if $U(\alpha f ; x) \neq|\alpha|^{2} U(f ; x)+V(\alpha, f, x)$.

\section{REFERENCE}

1. D. C. Youla, L. J. Castriota, and H. J. Carlin, Bounded real scattering matrices and the foundations of linear passive network theory, IRE PGCT, Vol. CT-6, March 1959. 